\title{
Spectral problems for fractional differential equations from nonlocal continuum mechanics
}

Jing Li and Jiangang Qi

Correspondence: qjg816@163.com School of Mathematics and Statistics, Shandong University, Weihai, Shandong 264209, P.R. China

\begin{abstract}
This paper studies the spectral problem of a class of fractional differential equations from nonlocal continuum mechanics. By applying the spectral theory of compact self-adjoint operators in Hilbert spaces, we show that the spectrum of this problem consists of only countable real eigenvalues with finite multiplicity and the corresponding eigenfunctions form a complete orthogonal system. Furthermore, we obtain the lower bound of the eigenvalues.
\end{abstract}

MSC: Primary 26A33; 34L15; secondary 34B10; 47E05

Keywords: fractional differential equation; self-adjoint; eigenfunction; eigenvalue problem

\section{Introduction}

The present paper studies the spectral problem associated to the fractional differential equation (FDE) with left and right fractional derivatives

$$
-y^{\prime \prime}+q(x) y+\mu\left(D_{0+}^{\alpha}+D_{1-}^{\alpha}\right) y=\lambda y \quad \text { on }(0,1)
$$

where $q$ is a real-valued function, $D_{0+}^{\alpha}$ and $D_{1-}^{\alpha}$ are the left and right Riemann-Liouville fractional derivatives of order $\alpha$, respectively, whose definitions are given later, $\mu$ is a real constant and $\lambda$ is the spectral parameter.

Recently, fractional differential equations have drawn much attention. It is caused both by the development of fractional calculus itself and by the applications in various fields of science and engineering such as control, electrochemistry, electromagnetic, porous media, and viscoelasticity. For details, see [1-5].

Fractional differential equations with both left and right fractional derivatives are also applicable to many fields, such as the extremal problems of fractional Euler-Lagrange equations $[6,7]$ and the optimal control theory for functionals involving fractional derivatives [8]. For the study of FDEs with left and right fractional derivatives, we mention the papers [9-12].

Sturm-Liouville Theory was the basis for the development of spectral theory of differential operators, which was profoundly influenced by the emergence of quantum mechanics in the early years of the twentieth century [13]. In the last few years, many problems in science and engineering can be modeled accurately in the form of fractional calculus such

\section{Springer}

@2014 Li and Qi; licensee Springer. This is an Open Access article distributed under the terms of the Creative Commons Attribution License (http://creativecommons.org/licenses/by/2.0), which permits unrestricted use, distribution, and reproduction in any medium, provided the original work is properly cited. 
as multi-scale problems that span a wide range of time or length scales. In many situations, it need to consider the spectral problems of FDEs in order to tackle these practical problems. Meanwhile, studying the spectral problems of FDEs is also very important for enriching and improving the theories of FDEs. The spectral problems of FDEs have been announced earlier [14-16], but they have not been thoroughly studied either qualitatively or quantitatively. In addition, numerical computing of the spectral problems of FDEs has attracted much research attention (see, e.g., [17-25]). However, these researches do not examine the common properties of the eigenvalues and eigenfunctions. Up till now, there are only a few papers on this topic (see [9-11]). Klimek and Agrawal $[10,11]$ investigated the fractional differential equations

$$
{ }^{C} D_{b-}^{\alpha} p(x) D_{a+}^{\alpha} f_{\lambda}(x)+q(x) f_{\lambda}(x)+\lambda \omega_{\alpha}(x) f_{\lambda}(x)=0
$$

and

$$
{ }^{C} D_{a+}^{\alpha} p(x) D_{b-}^{\alpha} f_{\lambda}(x)+q(x) f_{\lambda}(x)+\lambda \omega_{\alpha}(x) f_{\lambda}(x)=0 .
$$

They demonstrated that the eigenvalues of the above two fractional eigenvalue problems are real, and the eigenfunctions corresponding to different eigenvalues are orthogonal.

The fractional differential equation (1.1) arises from the nonlocal continuum mechanics [26-32]. It is a governing equilibrium equation of an elastic bar of finite length $L$ with long-range interactions among non-adjacent particles. In [32], the equilibrium equation of the bar may be written as

$$
\frac{d^{2} u(x)}{d x^{2}}+\frac{\kappa_{\alpha}}{2}\left\{\left(D_{0+}^{\alpha}+D_{L-}^{\alpha}\right) u(x)-\frac{\alpha(\alpha-1)^{2}}{\Gamma(2-\alpha)}\left[\frac{u(0)}{x^{\alpha}}+\frac{u(L)}{(L-x)^{\alpha}}\right]\right\}=-\frac{f(x)}{E},
$$

where $u(x)$ is the axial displacement of the bar at $x, f(x)$ is the longitudinal force per unit volume, $E$ is Young's modulus, $D_{0+}^{\alpha}$ and $D_{L-}^{\alpha}$ are the left and right Riemann-Liouville fractional derivatives of order $\alpha(1<\alpha<2)$, respectively, and $\kappa_{\alpha}$ is a material constant. In a recent work, Qi and Chen [9] studied analytically the eigenvalue problem of order $\alpha \in(0,1)$ associated to the equation (1.1). They also proposed that the spectral problem associated to (1.1) with $1<\alpha<2$ is more interesting. The reason is that (1.1) with $1<\alpha<2$ can overcome some mechanical inconsistencies arising when the order of fractional derivative is in the range $(0,1)$. Therefore, this paper will focus on the spectral problem which was proposed in [9].

In this study, by using the spectral theory of self-adjoint compact operators in Hilbert spaces, we prove that the spectrum of the spectral problem associated to (1.1) with $1<\alpha<$ $3 / 2$ consists of only countable real eigenvalues with finite multiplicity and the orthogonal completeness of the corresponding eigenfunction system in the Hilbert spaces. Furthermore, we obtain the lower bound of the eigenvalues.

The rest of the paper is organized as follows. In Section 2, we give some preliminary knowledge for fractional derivatives and the spectral theory of Sturm-Liouville problems that will be needed to develop this work. In Section 3, we obtain the main results of this paper. 


\section{Preliminaries}

\subsection{Fractional derivatives}

We will use the following properties of fractional integrals and derivatives which can be found in [3].

Definition $1(c f$. [3, p.69]) Let $R(\alpha)>0$. The left and right Riemann-Liouville integrals of order $\alpha$ are defined by

$$
\left(I_{0+}^{\alpha} f\right)(x)=\frac{1}{\Gamma(\alpha)} \int_{0}^{x} \frac{f(s)}{(x-s)^{1-\alpha}} d s, \quad x \in(0,1]
$$

and

$$
\left(I_{1-}^{\alpha} f\right)(x)=\frac{1}{\Gamma(\alpha)} \int_{x}^{1} \frac{f(s)}{(s-x)^{1-\alpha}} d s, \quad x \in[0,1),
$$

respectively, where $\Gamma$ denotes the Euler gamma function.

Definition $2(c f$. [3, p.70]) Let $R(\alpha) \in(n-1, n), D=d / d x$. The left and right RiemannLiouville derivatives of order $\alpha$ are defined by (when they exist)

$$
\left(D_{0+}^{\alpha} f\right)(x)=D^{n}\left(I_{0+}^{n-\alpha} f\right)(x), \quad x \in(0,1]
$$

and

$$
\left(D_{1-}^{\alpha} f\right)(x)=(-D)^{n}\left(I_{1-}^{n-\alpha} f\right)(x), \quad x \in[0,1),
$$

respectively. In particular, when $1<\alpha<2$, we have

$$
\left(D_{0+}^{\alpha} f\right)(x)=D^{2}\left(I_{0+}^{2-\alpha} f\right)(x)=\Gamma_{\alpha}\left(\int_{0}^{x} \frac{f(t)}{(x-t)^{\alpha-1}} d t\right)^{\prime \prime}, \quad x \in(0,1]
$$

and

$$
\left(D_{1-}^{\alpha} f\right)(x)=D^{2}\left(I_{1-}^{2-\alpha} f\right)(x)=\Gamma_{\alpha}\left(\int_{x}^{1} \frac{f(t)}{(t-x)^{\alpha-1}} d t\right)^{\prime \prime}, \quad x \in[0,1),
$$

where $\Gamma_{\alpha}=1 / \Gamma(2-\alpha)$.

Proposition 2.1 (cf. [3, Lemma 2.2, p.73]) Let $R(\alpha) \in(n-1, n)$. If $y(x) \in A C^{n}[0,1]$, then the fractional derivatives $D_{0_{+}}^{\alpha} y$ and $D_{1-}^{\alpha} y$ exist almost everywhere on $[0,1]$ and can be represented in the forms

$$
\left(D_{0+}^{\alpha} y\right)(x)=\sum_{k=0}^{n-1} \frac{y^{(k)}(0)}{\Gamma(1+k-\alpha)} x^{k-\alpha}+\frac{1}{\Gamma(n-\alpha)} \int_{0}^{x} \frac{y^{(n)}(t) d t}{(x-t)^{\alpha-n+1}}, \quad x \in(0,1]
$$

and

$$
\left(D_{1-}^{\alpha} y\right)(x)=\sum_{k=0}^{n-1} \frac{(-1)^{k} y^{(k)}(1)}{\Gamma(1+k-\alpha)}(1-x)^{k-\alpha}+\frac{(-1)^{n}}{\Gamma(n-\alpha)} \int_{x}^{1} \frac{y^{(n)}(t) d t}{(t-x)^{\alpha-n+1}}, \quad x \in[0,1) .
$$




\subsection{Eigenvalue problems for second order differential equations}

This subsection collects some known results from the spectral theory of second order differential equations with a view on the aim of this paper. Consider the regular SturmLiouville eigenvalue problem

$$
\tau y:=-y^{\prime \prime}+q y=\lambda y \quad \text { on }(0,1)
$$

subject to Dirichlet boundary conditions

$$
y(0)=0=y(1)
$$

where $q \in L(0,1)$ is a real-valued function. It is known that there exist countable simple real eigenvalues satisfying

$$
-\infty<\lambda_{1}<\lambda_{2}<\cdots<\lambda_{n}<\cdots, \quad n \rightarrow \infty .
$$

These results can be obtained by Prüfer transformation [33, Theorem 8.18].

Let $L^{2}=L^{2}(0,1)$ be the Hilbert space with the usual inner product $\langle f, g\rangle$ and the norm $\|f\|=\langle f, f\rangle^{1 / 2}$. Let $A C[0,1]$ be the set of all absolutely continuous, complex-valued functions on $[0,1]$. Consider the associated operator $T_{0}$ :

$$
\begin{aligned}
& \mathcal{D}\left(T_{0}\right)=\left\{y \in L^{2}: y, y^{\prime} \in A C[0,1], \tau y \in L^{2}, y(0)=y(1)=0\right\}, \\
& T_{0} y=\tau y, \quad y \in \mathcal{D}\left(T_{0}\right) .
\end{aligned}
$$

The operator $T_{0}$ is self-adjoint, or $T_{0}^{*}=T_{0}$, i.e.,

(i) the domain $\mathcal{D}\left(T_{0}\right)$ is dense in $L^{2}$;

(ii) $T_{0}$ is Hermitian, or $\langle T f, g\rangle=\langle f, T g\rangle$ for $f, g \in \mathcal{D}\left(T_{0}\right)$;

(iii) $\mathcal{D}\left(T_{0}\right)=\mathcal{D}\left(T_{0}^{*}\right)$, where $T_{0}^{*}$ is the adjoint of $T_{0}$.

For details of these definitions, the reader is referred to [34,35]. If 0 is not an eigenvalue of $T_{0}$, then there exists a Green function $G(x, t)$ defined by

$$
G(x, t)= \begin{cases}u(t) v(x), & 0 \leq t \leq x \leq 1 \\ u(x) v(t), & 0 \leq x \leq t \leq 1\end{cases}
$$

such that for $f \in L^{2}, y$ is a solution of $T_{0} y=f$ if and only if

$$
y(x)=\int_{0}^{1} G(x, t) f(t) d t,
$$

where $u, v$ are the solutions of $\tau y=0$ such that

$$
u(0)=0, \quad v(1)=0, \quad u v^{\prime}-u^{\prime} v \equiv-1 \quad \text { on }[0,1] .
$$

We also call the function $G$ the Green function associated to the operator $T_{0}$. 


\section{The fractional operator}

In this section, we will prove that the spectral problem of (1.1) with Dirichlet boundary value conditions has only countable real eigenvalues with finite multiplicity. Consider the spectral problem

$$
\left\{\begin{array}{l}
-y^{\prime \prime}(x)+q(x) y(x)+\mu\left(D_{0+}^{\alpha}+D_{1-}^{\alpha}\right) y(x)=\lambda y(x) \quad \text { on }(0,1) \\
y(0)=y(1)=0
\end{array}\right.
$$

where $D_{0_{+}}^{\alpha}$ and $D_{1-}^{\alpha}$ are the left and right Riemann-Liouville fractional derivatives of order $\alpha$, respectively, defined as in Definition 2. The fractional operator $T$ associated to (3.1) is defined by

$$
T y=T_{0} y+\mu\left(D_{0+}^{\alpha}+D_{1-}^{\alpha}\right) y, \quad y \in \mathcal{D}(T):=\mathcal{D}\left(T_{0}\right) .
$$

Throughout this paper we always assume that $q \in L^{2}(0,1)$ and $1<\alpha<3 / 2$.

The next lemma reveals the rationality of $1<\alpha<3 / 2$.

Lemma 3.1 If $q \in L^{2}$ and $1<\alpha<2$, then $T$ is a linear operator from $\mathcal{D}(T)$ to $L^{2}$ if and only if $1<\alpha<3 / 2$.

Proof Since $1<\alpha<2$, for $y \in \mathcal{D}(T)$, it follows from Definition 2 that $D_{1-}^{\alpha} y$ and $D_{0_{+}}^{\alpha} y$ exist. Note that $T_{0} y \in L^{2}$ for $y \in \mathcal{D}(T)$. Then $q \in L^{2}$ implies that $y^{\prime \prime} \in L^{2}$. By Proposition 2.1 and the boundary conditions $y(0)=0=y(1)$,

$$
\left(D_{0+}^{\alpha}+D_{1-}^{\alpha}\right) y(x)=\Gamma_{\alpha}\left[\int_{0}^{1} \frac{y^{\prime \prime}(t) d t}{|x-t|^{\alpha-1}}+\frac{y^{\prime}(0)}{x^{\alpha-1}}-\frac{y^{\prime}(1)}{(1-x)^{\alpha-1}}\right] .
$$

Using the Cauchy-Schwarz inequality and integrating by parts, we have

$$
\begin{aligned}
& \int_{0}^{1}\left|\int_{0}^{1} \frac{y^{\prime \prime}(t) d t}{|x-t|^{\alpha-1}}\right|^{2} d x \\
& \quad \leq \int_{0}^{1}\left(\int_{0}^{1}|x-t|^{1-\alpha} d t\right)\left(\int_{0}^{1} \frac{\left|y^{\prime \prime}(t)\right|^{2} d t}{|x-t|^{\alpha-1}}\right) d x \\
& \quad \leq \frac{2^{\alpha-1}}{2-\alpha} \int_{0}^{1}\left(\int_{0}^{1} \frac{\left|y^{\prime \prime}(t)\right|^{2} d t}{|x-t|^{\alpha-1}}\right) d x \\
& \quad=\frac{2^{\alpha-1}}{2-\alpha} \int_{0}^{1}\left(\int_{0}^{1}|x-t|^{1-\alpha} d x\right)\left|y^{\prime \prime}(t)\right|^{2} d t \leq\left(\frac{2^{\alpha-1}}{2-\alpha}\right)^{2}\left\|y^{\prime \prime}\right\|^{2} .
\end{aligned}
$$

Here we have used the fact that

$$
\int_{0}^{1}|x-t|^{1-\alpha} d t=\frac{x^{2-\alpha}+(1-x)^{2-\alpha}}{2-\alpha} \leq \frac{2^{\alpha-1}}{2-\alpha}, \quad \text { for } x \in[0,1] .
$$

Therefore $\int_{0}^{1}|x-t|^{1-\alpha} y^{\prime \prime}(t) d t \in L^{2}$.

Let $1<\alpha<3 / 2$, then $x^{1-\alpha},(1-x)^{1-\alpha} \in L^{2}$ by calculation, and hence $T y \in L^{2}$, which implies that $T$ is a linear operator from $\mathcal{D}(T)$ to $L^{2}$. 
Conversely, let $T$ be a linear operator from $\mathcal{D}(T)$ to $L^{2}$. Note that for any $y \in \mathcal{D}(T)$, we have $T_{0} y \in L^{2}$, which implies that $\left(D_{0+}^{\alpha}+D_{1-}^{\alpha}\right) y \in L^{2}$. It follows from $\left|\int_{0}^{1}\right| x-\left.t\right|^{1-\alpha} y^{\prime \prime}(t) d t \mid \in$ $L^{2}$ and (3.3) that, for any $y \in \mathcal{D}(T)$,

$$
\frac{y^{\prime}(0)}{x^{\alpha-1}}-\frac{y^{\prime}(1)}{(1-x)^{\alpha-1}} \in L^{2} .
$$

By the arbitrariness of $y$, we can choose $y \in \mathcal{D}(T)$ such that $y^{\prime}(0) \neq 0$ and $y^{\prime}(1)=0$. Then by (3.5), we have $x^{1-\alpha} \in L^{2}$, which implies that $1<\alpha<3 / 2$.

Lemma 3.2 $T$ is symmetric for $\mu \in \mathbb{R}$, i.e., $\mathcal{D}(T)$ is dense in $L^{2}$ and

$$
\langle T y, f\rangle=\langle y, T f\rangle, \quad f, y \in \mathcal{D}(T) .
$$

Proof Since $\mathcal{D}(T)=\mathcal{D}\left(T_{0}\right)$ and $\mathcal{D}\left(T_{0}\right)$ is dense in $L^{2}, \mathcal{D}(T)$ is also dense in $L^{2}$. Considering the symmetry of $T_{0}$, it suffices to prove that

$$
\int_{0}^{1} f(x)\left[\left(D_{0_{+}}^{\alpha}+D_{1-}^{\alpha}\right) \bar{y}\right](x) d x=\int_{0}^{1} \bar{y}(x)\left[\left(D_{0_{+}}^{\alpha}+D_{1-}^{\alpha}\right) f\right](x) d x, \quad \text { for } f, y \in \mathcal{D}(T) .
$$

For $y, f \in \mathcal{D}(T)$, by the definitions of $D_{0_{+}}^{\alpha}, D_{1_{-}}^{\alpha}$ and integrating by parts, we have

$$
\begin{aligned}
& \int_{0}^{1} f(x)\left(D_{0+}^{\alpha} \bar{y}\right)(x) d x \\
& =\Gamma_{\alpha} \int_{0}^{1} f(x) \cdot\left(\frac{d}{d x}\right)^{2}\left(\int_{0}^{x} \frac{\bar{y}(t)}{(x-t)^{\alpha-1}} d t\right) d x \\
& =-\Gamma_{\alpha} \int_{0}^{1} f^{\prime}(x)\left(\int_{0}^{x} \frac{\bar{y}(t)}{(x-t)^{\alpha-1}} d t\right)^{\prime} d x \\
& =-\Gamma_{\alpha} f^{\prime}(1) \int_{0}^{1} \frac{\bar{y}(t)}{(1-t)^{\alpha-1}} d t+\Gamma_{\alpha} \int_{0}^{1} f^{\prime \prime}(x)\left(\int_{0}^{x} \frac{\bar{y}(t)}{(x-t)^{\alpha-1}} d t\right) d x \\
& =-\Gamma_{\alpha} f^{\prime}(1) \int_{0}^{1} \frac{\bar{y}(t)}{(1-t)^{\alpha-1}} d t+\Gamma_{\alpha} \int_{0}^{1}\left(\int_{t}^{1} \frac{f^{\prime \prime}(x)}{(x-t)^{\alpha-1}} d x\right) \bar{y}(t) d t \\
& =-\Gamma_{\alpha} f^{\prime}(1) \int_{0}^{1} \frac{\bar{y}(t)}{(1-t)^{\alpha-1}} d t+\int_{0}^{1}\left[\left(D_{1-}^{\alpha} f\right)(t)+\Gamma_{\alpha} f^{\prime}(1)(1-t)^{1-\alpha}\right] \bar{y}(t) d t \\
& =\int_{0}^{1} \bar{y}(x)\left(D_{1-}^{\alpha} f\right)(x) d x .
\end{aligned}
$$

Here we have used $f(1)=0=y(1)$ and exchanged the order of integration. Similarly, we can prove that

$$
\int_{0}^{1} f(x)\left(D_{1-}^{\alpha} \bar{y}\right)(x) d x=\int_{0}^{1} \bar{y}(x)\left(D_{0+}^{\alpha} f\right)(x) d x
$$

and hence $T$ is symmetric.

We will use the following result to prove the self-adjointness of $T$. 
Lemma 3.3 ( $c f$. [35, Theorem 5.19, p.107]) If for some $\lambda_{0} \in \mathbb{R}$, the range $\mathcal{R}\left(T-\lambda_{0}\right)$ of $T-\lambda_{0}$ is the entire space $L^{2}$, then $T$ is self-adjoint.

In order to apply Lemma 3.3, we first prepare three lemmas.

Lemma 3.4 Let $\lambda_{1}$ be the first eigenvalue of $T_{0}$. Choose $\lambda_{0}<\lambda_{1}$. Then for $f \in L^{2},\left(T-\lambda_{0}\right) y=$ $f$ can be rewritten as the integral equation

$$
y=K y+F, \quad K y=\int_{0}^{1} K(\cdot, t) y(t) d t, \quad F=\int_{0}^{1} G(\cdot, t) f(t) d t,
$$

where

$$
\begin{aligned}
& K(x, t)=\mu \Gamma_{\alpha}\left[-K_{1}(x, t)-K_{2}(x, t)-\frac{1}{|x-t|^{\alpha-1}}+\frac{v(x) u^{\prime}(0)}{t^{\alpha-1}}-\frac{u(x) v^{\prime}(1)}{(1-t)^{\alpha-1}}\right], \\
& K_{1}(x, t)= \begin{cases}\int_{t}^{x} \frac{v(x) u^{\prime \prime}(s)}{(s-t) \alpha-1} d s+\int_{x}^{1} \frac{u(x) v^{\prime \prime}(s)}{(s-t)^{\alpha-1}} d s, & 0 \leq t \leq x \leq 1, \\
\int_{t}^{1} \frac{u(x) v^{\prime \prime}(s)}{(s-t)^{\alpha-1}} d s, & 0 \leq x \leq t \leq 1,\end{cases} \\
& K_{2}(x, t)= \begin{cases}\int_{0}^{t} \frac{v(x) u^{\prime \prime}(s)}{(t-s) \alpha-1} d s, & 0 \leq t \leq x \leq 1, \\
\int_{0}^{x} \frac{v(x) u^{\prime \prime}(s)}{(t-s)^{\alpha-1}} d s+\int_{x}^{t} \frac{u(x) v^{\prime \prime}(s)}{(t-s)^{\alpha-1}} d s, & 0 \leq x \leq t \leq 1,\end{cases}
\end{aligned}
$$

and $u(x), v(x)$ are the solutions of $T_{0} y=0$ such that $u(0)=0, v(1)=0, u v^{\prime}-u^{\prime} v \equiv-1$ on $[0,1]$.

Proof By the definition of $\lambda_{1}$ and $\lambda_{0}<\lambda_{1}$, we know that 0 is not an eigenvalue of $T_{0}-\lambda_{0}$. Thus it follows from (2.10) that $y$ is a solution of $\left(T-\lambda_{0}\right) y=f$ if and only if

$$
y(x)=\int_{0}^{1} G(x, t)\left[f(t)-\mu\left(D_{0_{+}}^{\alpha}+D_{1-}^{\alpha}\right) y(t)\right] d t,
$$

where $G$ is the Green function associated to $T_{0}-\lambda_{0}$. A calculation gives

$$
-\mu \int_{0}^{1} G(x, t)\left(D_{0_{+}}^{\alpha}+D_{1-}^{\alpha}\right) y(t) d t=\int_{0}^{1} K(x, t) y(t) d t
$$

where $K(x, t)$ is defined as (3.9). Therefore

$$
y(x)=\int_{0}^{1} K(x, t) y(t) d t+\int_{0}^{1} G(x, t) f(t) d t=K y+F .
$$

Remark 1 The details of the computation of (3.11) are presented in the appendix.

Lemma 3.5 If $|\mu|<\Gamma(3-\alpha) / 2$, then $T$ is self-adjoint.

Proof Step 1. We prove that

$$
\left|\left\langle\left(D_{0_{+}}^{\alpha}+D_{1-}^{\alpha}\right) y, y\right\rangle\right| \leq \frac{2\left\|y^{\prime}\right\|^{2}}{\Gamma(3-\alpha)}, \quad \text { for } y \in \mathcal{D}(T) .
$$


It follows from the definition of $D_{0+}^{\alpha}$ and integrating by parts that

$$
\begin{aligned}
\left|\left\langle D_{0+}^{\alpha} y, y\right\rangle\right| & =\left|\int_{0}^{1} y(x) \cdot\left(D_{0+}^{\alpha} \bar{y}\right)(x) d x\right|=\Gamma_{\alpha}\left|\int_{0}^{1} y(x) \cdot\left(\frac{d}{d x}\right)^{2}\left(\int_{0}^{x} \frac{\bar{y}(t)}{(x-t)^{\alpha-1}} d t\right) d x\right| \\
& =\Gamma_{\alpha}\left|\int_{0}^{1} y^{\prime}(x)\left(\int_{0}^{x} \frac{\bar{y}(t)}{(x-t)^{\alpha-1}} d t\right)^{\prime} d x\right|=\Gamma_{\alpha}\left|\int_{0}^{1} y^{\prime}(x)\left(\int_{0}^{x} \frac{\bar{y}^{\prime}(t)}{(x-t)^{\alpha-1}} d t\right) d x\right| \\
& \leq \Gamma_{\alpha}\left(\int_{0}^{1}\left|y^{\prime}(x)\right|^{2} d x\right)^{1 / 2}\left(\int_{0}^{1}\left|\int_{0}^{x} \frac{\bar{y}^{\prime}(t) d t}{(x-t)^{\alpha-1}}\right|^{2} d x\right)^{1 / 2} \\
& \leq \Gamma_{\alpha}\left\|y^{\prime}\right\|\left[\int_{0}^{1}\left(\int_{0}^{x}(x-t)^{1-\alpha} d t\right)\left(\int_{0}^{x} \frac{\left|\bar{y}^{\prime}(t)\right|^{2} d t}{(x-t)^{\alpha-1}}\right) d x\right]^{1 / 2} \\
& \leq \frac{\Gamma_{\alpha}\left\|y^{\prime}\right\|}{\sqrt{2-\alpha}}\left[\int_{0}^{1}\left(\int_{t}^{1}(x-t)^{1-\alpha} d x\right)\left|\bar{y}^{\prime}(t)\right|^{2} d t\right]^{1 / 2} \\
& \leq \frac{\Gamma_{\alpha}\left\|y^{\prime}\right\|^{2}}{2-\alpha}=\frac{\left\|y^{\prime}\right\|^{2}}{\Gamma(3-\alpha)}, \quad \text { for } y \in \mathcal{D}(T) .
\end{aligned}
$$

Here we have used $y(0)=0=y(1)$, the Cauchy-Schwarz inequality and exchanged the order of integration. Using a similar method as in proving (3.13), we have

$$
\left|\left\langle D_{1-}^{\alpha} y, y\right\rangle\right| \leq \frac{\left\|y^{\prime}\right\|^{2}}{\Gamma(3-\alpha)}, \quad \text { for } y \in \mathcal{D}(T)
$$

A combination of (3.13) and (3.14) gives (3.12).

Step 2. We prove that if $|\mu|<\Gamma(3-\alpha) / 2$, then there exists $\lambda_{0}<\lambda_{1}$ such that for $y \in \mathcal{D}(T)$, $\left\langle\left(T-\lambda_{0}\right) y, y\right\rangle \geq\|y\|^{2}$.

Let $Q(x)=\int_{0}^{x} q(t) d t, Q_{0}=\max _{x \in[0,1]}\{|Q(x)|\}$ and $\epsilon=\frac{\Gamma(3-\alpha)-2|\mu|}{2 \Gamma(3-\alpha)}$. Integrating $y^{\prime}(x) \bar{y}^{\prime}(x)$ on $[0,1]$ by parts, and using $y(0)=y(1)=0$ and $T_{0} y=-y^{\prime \prime}+q y$, we have

$$
\left\|y^{\prime}\right\|^{2}=\int_{0}^{1}\left|y^{\prime}\right|^{2}=\int_{0}^{1} y\left(T_{0} \bar{y}-q \bar{y}\right)=\left\langle T_{0} y, y\right\rangle-\langle q y, y\rangle,
$$

where

$$
|\langle q y, y\rangle| \leq 2 Q_{0} \int_{0}^{1}\left|y^{\prime}\right||y| \leq \epsilon\left\|y^{\prime}\right\|^{2}+\frac{Q_{0}^{2}}{\epsilon}\|y\|^{2} .
$$

Then (3.15) and (3.16) give

$$
\left\langle T_{0} y, y\right\rangle=\left\|y^{\prime}\right\|^{2}+\int_{0}^{1} q|y|^{2} \geq(1-\epsilon)\left\|y^{\prime}\right\|^{2}-\frac{Q_{0}^{2}}{\epsilon}\|y\|^{2} .
$$

A combination of (3.12) and (3.17) gives

$$
\begin{aligned}
\left\langle\left(T-\lambda_{0}\right) y, y\right\rangle & =\left\langle T_{0} y, y\right\rangle+\mu\left\langle\left(D_{0_{+}}^{\alpha}+D_{1-}^{\alpha}\right) y, y\right\rangle-\lambda_{0}\|y\|^{2} \\
& \geq(1-\epsilon)\left\|y^{\prime}\right\|^{2}-\frac{Q_{0}^{2}}{\epsilon}\|y\|^{2}-\frac{2|\mu|}{\Gamma(3-\alpha)}\left\|y^{\prime}\right\|^{2}-\lambda_{0}\|y\|^{2} \\
& =\frac{\Gamma(3-\alpha)-2|\mu|}{2 \Gamma(3-\alpha)}\left\|y^{\prime}\right\|^{2}+\left(-\frac{2 \Gamma(3-\alpha) Q_{0}^{2}}{\Gamma(3-\alpha)-2|\mu|}-\lambda_{0}\right)\|y\|^{2} .
\end{aligned}
$$


Note that $|\mu|<\Gamma(3-\alpha) / 2$. Then

$$
\left\langle\left(T-\lambda_{0}\right) y, y\right\rangle \geq\|y\|^{2}
$$

for

$$
\lambda_{0} \leq \min \left\{-\frac{2 \Gamma(3-\alpha) Q_{0}^{2}}{\Gamma(3-\alpha)-2|\mu|}-1, \lambda_{1}\right\} .
$$

Step 3. We prove that $T$ is self-adjoint for $|\mu|<\Gamma(3-\alpha) / 2$.

Let $\lambda_{0}$ be defined as in (3.19). It follows from Lemma 3.4 that $y$ is a solution of $\left(T-\lambda_{0}\right) y=$ $f, f \in L^{2}$ if and only if $y=K y+F$, where $K$ and $F$ are defined as in (3.8) and (3.9), respectively. From the definition of $K(x, t)$, we conclude that $K(x, t)$ is continuous on $[0,1] \times[0,1]$. Hence the operator $K$ is compact and (3.8) is a Fredholm integral equation of the first kind. Note that $F \in L^{2}$ for $f \in L^{2}$ by the definition of $F$. If $y \in \mathcal{D}(T)$ is a solution of $\left(T-\lambda_{0}\right) y=0$, then (3.18) implies $y=0$, and hence $y=K y$ has only zero solution in $L^{2}$. It follows from the Fredholm Alternative Theorem that the equation $y=K y+F$ has a unique solution, which implies that $\left(T-\lambda_{0}\right) y=f$ for $f \in L^{2}$ has a unique solution. By the arbitrariness of $f$, we know that $R\left(T-\lambda_{0}\right)=L^{2}$, and hence $T$ is self-adjoint by Lemma 3.3.

Lemma 3.6 Let $\lambda_{0}$ be defined as in (3.19). If $|\mu|<\Gamma(3-\alpha) / 2$, then the resolvent $R\left(T, \lambda_{0}\right):=$ $\left(T-\lambda_{0}\right)^{-1}$ is well defined, self-adjoint, compact and positive, i.e. $\left\langle\left(T-\lambda_{0}\right) y, y\right\rangle>0$ for $0 \neq$ $y \in \mathcal{D}(T)$.

Proof The existence and self-adjointness of $R\left(T, \lambda_{0}\right)$ follow from the discussion in Lemma 3.5. The positivity follows from (3.18). It remains to prove that $R\left(T, \lambda_{0}\right)$ is compact. Let $f_{n}$ be a bounded sequence in $L^{2}$, say, $\left\|f_{n}\right\| \leq 1$ for $n \geq 1$. Set $y_{n}=R\left(T, \lambda_{0}\right) f_{n}$, or $\left(T-\lambda_{0}\right) y_{n}=f_{n}$. Then (3.18) gives $\left\|y_{n}\right\|^{2} \leq\left\langle\left(T-\lambda_{0}\right) y_{n}, y_{n}\right\rangle=\left\langle f_{n}, y_{n}\right\rangle \leq\left\|y_{n}\right\|, n \geq 1$, and hence $\left\|y_{n}\right\| \leq 1$. Since $K$ is compact in $L^{2},\left\{K y_{n}\right\}$ possesses a convergent subsequence, denoted by $\left\{K y_{n}\right\}$ again for simplicity. Since $G$ is also compact, we can find a convergent subsequence $\left\{F_{n_{k}}\right\}$ of $F_{n}=\left\{G f_{n}\right\}$ in $L^{2}$. Therefore, (3.8) means that $y_{n_{k}}=K y_{n_{k}}+F_{n_{k}}$ is convergent in $L^{2}$. Thus, $R\left(T, \lambda_{0}\right)$ is compact.

The following theorem is the main result of this paper.

Theorem 3.7 Let $q \in L^{2}, 1<\alpha<3 / 2$ and $|\mu|<\Gamma(3-\alpha) / 2$, then the eigenvalue problem (1.1) has countable real eigenvalues $\lambda_{n}$ with finite multiplicity, $n \geq 1$, such that

$$
-\infty<\lambda_{1} \leq \lambda_{2} \leq \cdots \leq \lambda_{n} \leq \cdots, \quad \lambda_{n} \rightarrow \infty, n \rightarrow \infty
$$

and the set of all corresponding eigenfunctions $\phi_{n}(x), n \geq 1$, forms a complete, orthogonal system of $L^{2}$. Moreover, the first eigenvalue $\lambda_{1}$ satisfies $\lambda_{1} \geq-\frac{2 \Gamma(3-\alpha) Q_{0}^{2}}{\Gamma(3-\alpha)-2|\mu|}$.

Proof From the spectral theorem of compact self-adjoint operators in Hilbert spaces we conclude that $R\left(T, \lambda_{0}\right)$ has only countable real eigenvalues $\xi_{n}$ with finite multiplicity, $n \geq 1$. Since $R\left(T, \lambda_{0}\right)$ is positive, its eigenvalues satisfy

$$
\xi_{1} \geq \xi_{2} \geq \cdots \geq \xi_{n} \geq \cdots>0 \quad \text { and } \quad \xi_{n} \rightarrow 0+, \quad n \rightarrow \infty
$$


Moreover, the eigenfunctions $\phi_{n}(x)$ of $R\left(T, \lambda_{0}\right)$ form a complete, orthogonal system of $L^{2}$. Note that the eigenvalues $\lambda_{n}$ of $T$ and $\xi_{n}$ of $R\left(T, \lambda_{0}\right)$ have the relations

$$
\lambda_{n}=\lambda_{0}+\frac{1}{\xi_{n}}, \quad n \geq 1
$$

The first part of this theorem follows from (3.21). We now prove $\lambda_{1} \geq-\frac{2 \Gamma(3-\alpha) Q_{0}^{2}}{\Gamma(3-\alpha)-2|\mu|}$ if $|\mu|<$ $\Gamma(3-\alpha) / 2$. In fact, by (3.12) and (3.17), we have

$$
\begin{aligned}
\lambda_{1}\|y\|^{2} & =\left\langle\lambda_{1} y, y\right\rangle=\langle T y, y\rangle=\left\langle T_{0} y, y\right\rangle+\mu\left\langle\left(D_{0_{+}}^{\alpha}+D_{1-}^{\alpha}\right) y, y\right\rangle \\
& \geq \frac{\Gamma(3-\alpha)-2|\mu|}{2 \Gamma(3-\alpha)}\left\|y^{\prime}\right\|^{2}-\frac{2 \Gamma(3-\alpha) Q_{0}^{2}}{\Gamma(3-\alpha)-2|\mu|}\|y\|^{2} \geq-\frac{2 \Gamma(3-\alpha) Q_{0}^{2}}{\Gamma(3-\alpha)-2|\mu|}\|y\|^{2},
\end{aligned}
$$

which implies $\lambda_{1} \geq-\frac{2 \Gamma(3-\alpha) Q_{0}^{2}}{\Gamma(3-\alpha)-2|\mu|}$ for $|\mu|<\Gamma(3-\alpha) / 2$.

\section{Appendix: Calculation of Lemma 3.4}

Let $\Gamma_{\alpha}=1 / \Gamma(2-\alpha)$. It follows from the definitions of $G(x, t), D_{0+}^{\alpha}$ and $D_{1-}^{\alpha}$ that

$$
\begin{aligned}
& \int_{0}^{1} G(x, t)\left(D_{0+}^{\alpha}+D_{1-}^{\alpha}\right) y(t) d t \\
& =\int_{0}^{x} u(t) v(x)\left(D_{0+}^{\alpha}+D_{1-}^{\alpha} y\right)(t) d t+\int_{x}^{1} u(x) v(t)\left(D_{0+}^{\alpha}+D_{1-}^{\alpha} y\right)(t) d t \\
& =\Gamma_{\alpha} \int_{0}^{x} u(t) v(x)\left(\frac{d}{d t}\right)^{2}\left(\int_{0}^{t} \frac{y(s) d s}{(t-s)^{\alpha-1}}\right) d t \\
& \quad+\Gamma_{\alpha} \int_{x}^{1} u(x) v(t)\left(\frac{d}{d t}\right)^{2}\left(\int_{0}^{t} \frac{y(s) d s}{(t-s)^{\alpha-1}}\right) d t \\
& \quad+\Gamma_{\alpha} \int_{0}^{x} u(t) v(x)\left(\frac{d}{d t}\right)^{2}\left(\int_{t}^{1} \frac{y(s) d s}{(s-t)^{\alpha-1}}\right) d t \\
& \quad+\Gamma_{\alpha} \int_{x}^{1} u(x) v(t)\left(\frac{d}{d t}\right)^{2}\left(\int_{t}^{1} \frac{y(s) d s}{(s-t)^{\alpha-1}}\right) d t .
\end{aligned}
$$

Integrating by parts and changing the order of integration, we have

$$
\begin{array}{rl}
\int_{0}^{x} & u(t) v(x)\left(\frac{d}{d t}\right)^{2}\left(\int_{0}^{t} \frac{y(s) d s}{(t-s)^{\alpha-1}}\right) d t \\
= & v(x)\left[u(x) \int_{0}^{x} \frac{y^{\prime}(s)}{(x-s)^{\alpha-1}} d s-\int_{0}^{x} u^{\prime}(t)\left(\int_{0}^{t} \frac{y(s) d s}{(t-s)^{\alpha-1}} d s\right)^{\prime} d t\right] \\
= & v(x)\left[u(x) \int_{0}^{x} \frac{y^{\prime}(s)}{(x-s)^{\alpha-1}} d s-u^{\prime}(x) \int_{0}^{x} \frac{y(s)}{(x-s)^{\alpha-1}} d s\right. \\
& \left.+\int_{0}^{x}\left(\int_{s}^{x} \frac{u^{\prime \prime}(t) d t}{(t-s)^{\alpha-1}}\right) y(s) d s\right] \\
\int_{x}^{1} & u(x) v(t)\left(\frac{d}{d t}\right)^{2}\left(\int_{0}^{t} \frac{y(s) d s}{(t-s)^{\alpha-1}}\right) d t \\
= & u(x)\left[-v(x) \int_{0}^{x} \frac{y^{\prime}(s) d s}{(x-s)^{\alpha-1}}-\int_{x}^{1} v^{\prime}(t)\left(\int_{0}^{t} \frac{y(s) d s}{(t-s)^{\alpha-1}}\right)^{\prime} d t\right]
\end{array}
$$




$$
\begin{aligned}
\int_{0}^{x} u(t) v(x)\left(\frac{d}{d t}\right)^{2}\left(\int_{t}^{1} \frac{y(s) d s}{(s-t)^{\alpha-1}}\right) d t \\
=v(x)\left[u(x) \int_{x}^{1} \frac{y^{\prime}(s) d s}{(s-x)^{\alpha-1}}-\int_{0}^{x} u^{\prime}(t)\left(\int_{t}^{1} \frac{y(s) d s}{(s-t)^{\alpha-1}}\right)^{\prime} d t\right] \\
=v(x)\left[u(x) \int_{x}^{1} \frac{y^{\prime}(s) d s}{(s-x)^{\alpha-1}}-u^{\prime}(x) \int_{x}^{1} \frac{y(s) d s}{(s-x)^{\alpha-1}}+u^{\prime}(0) \int_{0}^{1} \frac{y(s) d s}{s^{\alpha-1}}\right. \\
\left.\quad+\int_{0}^{x}\left(\int_{0}^{s} \frac{u^{\prime \prime}(t) d t}{(s-t)^{\alpha-1}}\right) y(s) d s+\int_{x}^{1}\left(\int_{0}^{x} \frac{u^{\prime \prime}(t) d t}{(s-t)^{\alpha-1}}\right) y(s) d s\right]
\end{aligned}
$$

and

$$
\begin{aligned}
\int_{x}^{1} u(x) v(t)\left(\frac{d}{d t}\right)^{2}\left(\int_{t}^{1} \frac{y(s) d s}{(s-t)^{\alpha-1}}\right) d t \\
=u(x)\left[-v(x) \int_{x}^{1} \frac{y^{\prime}(s) d s}{(s-x)^{\alpha-1}}-\int_{x}^{1} v^{\prime}(t)\left(\int_{t}^{1} \frac{y(s) d s}{(s-t)^{\alpha-1}}\right)^{\prime} d t\right] \\
=u(x)\left[-v(x) \int_{x}^{1} \frac{y^{\prime}(s) d s}{(s-x)^{\alpha-1}}+v^{\prime}(x) \int_{x}^{1} \frac{y(s) d s}{(s-x)^{\alpha-1}}\right. \\
\left.\quad+\int_{x}^{1}\left(\int_{x}^{s} \frac{v^{\prime \prime}(t) d t}{(s-t)^{\alpha-1}}\right) y(s) d s\right] .
\end{aligned}
$$

Combining (A.1) with (A.2)-(A.5) gives

$$
\begin{aligned}
-\mu & \int_{0}^{1} G(x, t)\left(D_{0+}^{\alpha}+D_{1-}^{\alpha} y\right)(t) d t \\
= & -\mu \Gamma_{\alpha}\left[\int_{0}^{x}\left(\int_{t}^{x} \frac{v(x) u^{\prime \prime}(s) d s}{(s-t)^{\alpha-1}}+\int_{x}^{1} \frac{u(x) v^{\prime \prime}(s) d s}{(s-t)^{\alpha-1}}\right) y(t) d t\right. \\
& +\int_{x}^{1}\left(\int_{t}^{1} \frac{u(x) v^{\prime \prime}(s) d s}{(s-t)^{\alpha-1}}\right) y(t) d t \\
& +\int_{0}^{x}\left(\int_{0}^{t} \frac{v(x) u^{\prime \prime}(s) d s}{(t-s)^{\alpha-1}}\right) y(t) d t+\int_{x}^{1}\left(\int_{0}^{x} \frac{v(x) u^{\prime \prime}(s) d s}{(t-s)^{\alpha-1}}+\int_{x}^{t} \frac{u(x) v^{\prime \prime}(s) d s}{(t-s)^{\alpha-1}}\right) y(t) d t \\
& \left.-\left(\int_{0}^{x} \frac{y(t) d t}{(x-t)^{\alpha-1}}+\int_{x}^{1} \frac{y(t) d t}{(t-x)^{\alpha-1}}\right)+\int_{0}^{1} \frac{v(x) u^{\prime}(0)}{t^{\alpha-1}} y(t) d t-\int_{0}^{1} \frac{u(x) v^{\prime}(1)}{(1-t)^{\alpha-1}} y(t) d t\right] \\
= & \int_{0}^{1} K(x, t) y(t) d t,
\end{aligned}
$$

where $K(x, t)$ is defined as in (3.9).

Competing interests

The authors declare that there is no conflict of interests regarding the publication of this article. 


\section{Acknowledgements}

The authors wish to thank the anonymous referees for providing valuable comments and suggestions which improved this paper. This research was partially supported by the National NSF of China (Grants 11271229, 11201263), the NSF of Shandong Province (Grants ZR2012AM002, ZR2012AQ004) and Independent Innovation Foundation of Shandong University (IIFSDU), China.

\section{Received: 19 November 2013 Accepted: 26 February 2014 Published: 13 Mar 2014}

\section{References}

1. Hilfer, R: Applications of Fractional Calculus in Physics. World Scientific, Singapore (2000)

2. Magin, RL: Fractional Calculus in Bioengineering. Begell House Inc., Redding (2006)

3. Kilbas, AA, Srivastava, HM, Trujillo, JJ: Theory and Applications of Fractional Differential Equations. Elsevier, Amsterdam (2006)

4. Bǎleanu, D, Diethelm, K Scalas, E, Trujillo, JJ: Fractional Calculus Models and Numerical Methods. Series on Complexity, Nonlinearity and Chaos. World Scientific, Boston (2012)

5. Mainardi, F: Fractional Calculus and Waves in Linear Viscoelasticity. Imperial College Press, London (2010)

6. Stanković, B: An equations in left and right fractional derivatives of the same order. Bull. Acad. Serbe Sci. Arts, Cl. Sci. Math. Nat., Sci. Nat. 33, 83-90 (2008)

7. Muslih, SI, Baleanu, D: Hamiltonian formulation of systems with linear velocities within Riemann-Liouville fractional derivatives. J. Math. Anal. Appl. 304, 599-606 (2005)

8. Frederico, GS, Torres, DFM: A formulation of Noether's theorem for fractional problems of the calculus variations. J. Math. Anal. Appl. 334, 834-846 (2007)

9. Qi, J, Chen, S: Eigenvalue problems of the model from nonlocal continuum mechanics. J. Math. Phys. 52, 073516 (2011)

10. Klimek, M, Agrawal, OP: On a regular fractional Sturm-Liouville problem with derivatives of order in $(0,1)$. In: Proceedings of the 13th International Carpathian Control Conference, Vysoke Tatry (Podbanske), Slovakia, pp. 28-31 (2012)

11. Klimek, M, Agrawal, OP: Fractional Sturm-Liouville problem. Comput. Math. Appl. (2013). doi:10.1016/j.camwa.2012.12.011

12. Atanackovic, TM, Stankovic, B: On a differential equation with left and right fractional derivatives. Fract. Calc. Appl. Anal. 10, 138-150 (2007)

13. Amrein, WO, Hinz, AM, Pearson, DB (eds.): Sturm-Liouville Theory. Past and Present. Birkhäuser, Basel (2005)

14. Flandrin, P: On the spectrum of fractional Brownian motions. IEEE Trans. Inf. Theory 35, 197-199 (1989)

15. He, XF: Fractional dimensionality and fractional derivatives spectral of interband optical transitions. Phys. Rev. B 42, $11751-11756(1990)$

16. Laskin, N: Fractional Schrödinger equation. Phys. Rev. E 66, 056108 (2002)

17. Baleanu, D, Bhrawy, AH, Taha, TM: Two efficient generalized Laguerre spectral algorithms for fractional initial value problems. Abstr. Appl. Anal. 2013, Article ID 546502 (2013)

18. Baleanu, D, Bhrawy, AH, Taha, TM: A modified generalized Laguerre spectral method for fractional differential equations on the half line. Abstr. Appl. Anal. 2013, Article ID 413529 (2013)

19. Jafarian, A, Ghaderi, P, Golmanichaneh, AK, Baleanu, D: On a one-dimensional nonlinear coupled system of equations in the theory of thermoelasticity. Rom. J. Phys. 58, 694-702 (2013)

20. Anwar, AMO, Jarad, F, Baleanu, D, Ayaz, F: Fractional Caputo heat equation within the double Laplace transform. Rom J. Phys. 58, 15-22 (2013)

21. Baleanu, D, Muslih, SI, Tas, K: Fractional Hamiltonian analysis of higher order derivatives systems. J. Math. Phys. 47, 103503 (2006). doi:10.1063/1.2356797

22. Baleanu, D: Fractional Hamiltonian analysis of irregular systems. Signal Process. 86, 2632-2636 (2006)

23. Ertürk, VS: Computing eigenelements of Sturm-Liouville problems of fractional order via fractional differential transform method. Math. Comput. Appl. 16(3), 712-720 (2011)

24. Jin, B, Runclell, W: An inverse Sturm-Liouville problem with a fractional derivative. J. Comput. Phys. 231, $4954-4966$ (2012)

25. Duan, JS, Wang, Z, Liu, YL, Qiu, X: Eigenvalue problems for fractional ordinary differential equations. Chaos Solitons Fractals 46, 46-53 (2013)

26. Cottone, G, Di Paola, M, Zingales, M: Dynamics of non-local systems handled by fractional calculus. In: 6th WESEAS International Conference, Cairo, Egypt, Dec. 29-31, pp. 81-89 (2007)

27. Di Paola, M, Zingales, M: Non-local continuum: fractional calculus approach. In: Proceeding of the XVIII AIMETA, Brescia, pp. 11-16 (2007)

28. Di Paola, M, Zingales, M: Long-range cohesive interactions of non-local continuum mechanics faced by fractional calculus. Int. J. Solids Struct. 45, 5642-5659 (2008)

29. Lazopoulos, KA: Non-local continuum mechanics and fractional calculus. Mech. Res. Commun. 33, $751-757$ (2006)

30. Polizzotto, C, Fuschi, P, Pisano, AA: A nonhomogeneous nonlocal elasticity model. Eur. J. Mech. A, Solids 25, 308-333 (2006)

31. Atanackovic, TM, Stankovic, B: Generalized wave equation in nonlocal elasticity. Acta Mech. 208, 1-10 (2009)

32. Carpinteri, A, Cornetti, P, Sapora, A: A fractional calculus approach to nonlocal elasticity. Eur. Phys. J. Spec. Top. 193, 193-204 (2011)

33. Hille, E: Lectures on Ordinary Differential Equations. Addison-Wesley, London (1969)

34. Kato, T: Perturbation Theory for Linear Operator. Springer, New York (1966)

35. Weidmann, J: Linear Operators in Hilbert Spaces. Springer, New York (1980)

10.1186/1687-1847-2014-85

Cite this article as: $\mathrm{Li}$ and Qi: Spectral problems for fractional differential equations from nonlocal continuum mechanics. Advances in Difference Equations 2014, 2014:85 